\title{
PONENCIA \\ SWINT-DBP: SOFTWARE INTEGRADOR PARA \\ EL DESARROLLO DE DESTREZAS BÁSICAS \\ DE PENSAMIENTO
}

\author{
Fernando Iriarte Díaz-Granados* \\ Isabel Sierra Pineda \\ Sara Noguera Hernández ${ }^{* * *}$
}

\begin{abstract}
The present work arises from the necessity of stimulating different the ways of learning which are required today in the con text of the global village, the society of knowledge and a technology-mediated universe. It also springs from the need for the enhancement of thinking skills which generate competencies that allow individuals to widen their fan of possibilities for human development and take advantage of the opportunity that offers the gradual incorporation of technologies to the curriculum. Taking ah this into account, a software was developed based on constructivist principies of íearning so that basic thinking skills in students of seventh grade are developed while working in an integrated way in the different curricular areas.
\end{abstract}

\section{RESUMEN}

El presente trabajo parte de la necesidad de estimular formas de aprendizaje que hoy son requeridas en el contexto de la globalización, la sociedad del conocimiento y el universo mediado con tecnologías; de la necesidad de enfatizar en las destrezas de pensamiento, cuyo conjunto genera competencias que permiten ampliar el abanico de posibilidades de desarrollo humano y de la oportunidad que ofrece la incorporación gradual de tecnologías al currículo. Por ello, se desarrollé un software basado en principios constructivistas del aprendizaje, que al tiempo que permitiera trabajar las áreas del currículo de una manera integrada, facilitara el desarrollo de destrezas básicas de pensamiento en alumnos de séptimo grado.

Palabras clave: integración de áreas, destrezas de pensamiento, constructivismo, software educativo.

\section{Presentación}

Ahora que en el nuevo contexto de la educación, gana cada día más importancia el enseñar a pensar, el esfuerzo investigativo que dio como resultado la producción de

\footnotetext{
* (firiarte@uninorte.edu.co) Universidad del Norte.

*** (edunexos2@yahoo.com) Universidad de Córdoba.

(snoguerah@hotmail.com) Universidad del Atlántico.
} 
SWINT-DBP, software integrador para el desarrollo de destrezas básicas de pensamiento, pone a disposición de educadores y alumnos de la educación básica, un material que aporta al tratamiento de contenidos curriculares desde la perspectiva de las destrezas básicas de pensamiento que un educando de secundaria debe dominar para el ejercicio permanente de sus habilidades cognitivas y la configuración de competencias en el saber pensar y saber hacer con sentido, a partir de la información proveniente de las áreas del conocimiento, apoyándose en mediaciones tecnológicas.

Se parte de la concepción de que las herramientas que provee el computador son importantes alternativas para mediar el aprendizaje siempre que hayan sido ideadas y diseñadas con enfoques y criterios adecuados en lo didáctico y lo pedagógico. En consecuencia, la tecnologíaa es ante todo "un recurso al servicio del educador y no un fin; como servicio a la docencia, la tecnología educativa coadyuva en los procesos de enseñanza y aprendizaje; es decir, el profesor se sirve de la tecnología educativa para enseñar y el estudiante para aprender y para socializar con sus colegas lo que aprende" (Alanis, A. 2000: 1).

¿Cómo y qué aspectos de la educabilidad del ser humano deben ser trabajadas con apoyo de la tecnología?, es el interrogante que guía la reflexión para configurar una pedagogía de la tecnología y con la tecnología, articulada en lo epistemológico, en lo procedimental y lo actitudinal, para que determine nuevos saberes y actuaciones en los docentes y los educandos en formación en los diferentes estadios de escolaridad en Colombia.

El ámbito del uso de mediaciones tecnológicas actúa como plataforma no solo para operacionalizar con sentido la integración curricular desde los contenidos conceptuales y metodológicos de las áreas, sino para favorecer en los educandos un nivel de control y conciencia sobre contenidos valorativos, implícitos o explícitos del currículo convencional. Según Miguel de Zubiría, "los conceptos corresponden a organizaciones internas de las nociones y las categorías son organizaciones externas interconceptuales", lo cual justifica que las actividades didácticas y los recursos instrumentales propuestas por el docente para el desarrollo de aprendizajes consistentes y significativos incentiven los procesos de atención hacia la construcción de nexos o vínculos entre conocimientos, desarrollo de habilidades en La aplicación de esos constructos y favorecer los afectos o valoraciones por el saber que se aprende (De Zubiría, M. 1995:134,152).

El programa SWINT pretende, a partir de estas premisas, aportar un modelo de trabajo metodológico que mediado por tecnologías de la información en la categoría de software educativo, estimule y desarrolle el pensamiento de los educandos en su nivel inicial en secundaria, partiendo de la integración de saberes desde su cotidianidad.

Con fundamento en la corriente constructivista de la enseñanza, el software se diseñó de manera que los aprendizajes y el conocimiento se construyera a partir de lo que el alumno "ya sabía" y navegara con sentido hacia lo que se requiere para su eficiente desenvolvimiento en lo cotidiano, haciendo del ejercicio del pensamiento, una expresión del potencial humano, coherente con los nuevos escenarios o ámbitos culturales, sociales y científicos en los que crecen y se desarrollan estos educandos. 
El software se enmarca conceptualmente dentro de las teorías cognitivas, específicamente del aprendizaje significativo y desde el punto de vista pedagógico se ubica en el constructivismo moderado (Gros, 1997: 82-83).

Dentro del constructivismo se identifican dos tendencias atendiendo a la posibilidad de especificar a priori el material a utilizar: el constructivismo radical llamado así por Merrill (1991), y denominado WIG por Perkins y el constructivismo moderado o constructivismo BIG (Perkins, 1991).

Las iniciales WIG provienen de without the information given. Sin información especificada. No hay límites en la información a utilizar. El alumno se puede basar en el material que le proporciona el formador y en materiales extraordinarios a los que acceda por sus propios medios o asesorado por el propio formador. Como se observa, los materiales no se definen a priori, sino que es el propio alumno el que marca los límites de la información a la que quiere acceder. El profesor guía el proceso sin dar respuestas, hay mucho de intuición aplicada por el que aprende para la búsqueda y construcción de modelos.

El constructivismo BIG obtiene su nombre de las iniciales de la frase en inglés beyond the information given. A través de la información dada. El constructivismo moderado, permite establecer parcialmente los contenidos a transmitir. Esta aproximación tiene elementos de instructivismo ya que el formador establece los límites de la información que va a utilizar el alumno. Aquí la construcción del significado es producto de la información propuesta a los educandos quienes aplican sus conocimientos a través de actividades realizadas con la información dada y es esa combinación la que se ha adoptado para el diseño del software como estrategia de desarrollo de destrezas de pensamiento en un nivel básico, con la diferencia de que su énfasis no es un contenido a transmitir sino que se vale de la información contenida en su base de datos para el ejercicio de operaciones sobre ese sustrato utilizando metáforas y escenarios de construcción, aplicación de los saberes previos para la solución de retos (Gros, 1997: 82-83).

La base de la discusión entre constructivistas moderados y radicales es la preespecificación externa de contenidos. El profesor que aplica enfoques moderados diseña entornos donde determina de antemano qué tareas son más relevantes para cada grupo de estudiantes y propone la estructura general del conocimiento, sin que ello signifique falta de flexibilidad.

El diseño pedagógico es la fortaleza de esta propuesta, y se evidencia con detalle en las descripciones y explicaciones, lo que facilita el desarrollo del material, determina la naturaleza de los componentes de su interfaz de navegación, las condiciones de aplicación del mismo con indicaciones precisas sobre cómo proceder para interactuar, y el tipo de respuesta que se debe esperar del programa frente a las acciones en cada actividad de los escenarios.

Este programa computacional se sitúa pedagógicamente en la metáfora de la construcción que enfatiza la importancia del entorno sobre los contenidos de aprendizaje. Desde la teoría constructivista de los aprendizajes, el contexto, lejos de ser instructivo, es más un entorno mediado por estrategias que involucran actividades cognitivas y contenidos que se podrán desarrollar y aprender con cierto nivel de libertad. El educando se sitúa desde lo que "ya sabe"; el programa le propone situaciones que en lugar de 
activar la memoria le permitan aplicar y vincular los conceptos previos con los nuevos, esto es, aprender significativamente, relacionando los nuevos contenidos con el conocimiento almacenado en sus esquemas mentales. Se trata, como lo indica Fogarty (1999) de construir intelectos creando condiciones para que el aprendizaje ocurra naturalmente y con sentido. En su concepto, desde la perspectiva de la pedagogía constructivista, esos arquitectos han sido: Dewey, Piaget, Vygotsky, Feuerstein, Gardner y Diamond.

\section{DISEÑO DEL SOFTWARE}

\section{Tipo de software}

El software es de carácter heurístico, es decir, caracterizado por un ambiente de aprendizaje abierto en el que los estudiantes llegan por experimentación al descubrimiento de lo que se desea que aprendan. Infiere el conocimiento, lo prueba y lo corrige si es el caso, creando así sus propios modelos de pensamiento respecto a lo que se está aprendiendo y desarrolla capacidades de autoaprendizaje.

Como ocurre en estos tipos de software, el alumno dispone de un micromundo, (en este caso la ciudad, el zoológico, el supermercado) compuesto por una serie de actividades (limpiando el parque, ayudando en la alcaldía, clasificando animales etc.) que se pueden manipular de acuerdo con un conjunto de reglas determinadas. En este caso no se le dice al usuario explícitamente lo que debe hacer sino que se le dan pistas que le permitan descubrir las reglas de trabajo.

\section{Descripción del contenido del software}

Tomando como punto de partida el problema formulado, así como la conducta de entrada y campo vital de la población objeto, se estableció lo que se debía enseñar o reforzar para responder a las necesidades encontradas, con apoyo del material computacional. Como resultado de la fase de diseño educativo, se obtuvo lo siguiente:

- Tipos de contenidos y destrezas asociadas.

- Estructura básica de la mediación.

- Sistema de motivación.

- Sistema de evaluación.

Las estrategias o actividades explícitas en el software se relacionan con contenidos conceptuales, metodológicos y valorativos, de las áreas de ciencias naturales, matemáticas, ciencias sociales y lenguaje.

— Despliegue de contenidos: 
Tabla 2. Contenidos del software.

\begin{tabular}{|c|c|c|c|c|}
\hline $\begin{array}{c}\text { Área } \\
\text { Tipos de } \\
\text { contenidos }\end{array}$ & Matemáticas & $\begin{array}{l}\text { Ciencias } \\
\text { Sociales }\end{array}$ & $\begin{array}{l}\text { Ciencias } \\
\text { Naturales }\end{array}$ & Lenguaje \\
\hline $\begin{array}{l}\text { Contenidos } \\
\text { conceptuales }\end{array}$ & $\begin{array}{l}\text { Propiedades de } \\
\text { los números } \\
\text { naturales, } \\
\text { conjuntos y } \\
\text { fraccionarios, } \\
\text { Sistema métrico, }\end{array}$ & \begin{tabular}{lrr}
\multicolumn{3}{c}{ Organización } \\
social, & medio \\
geográfico, & medio \\
natural y & cultural, \\
consumo & de \\
bienes & y \\
servicios. & & \\
Funciones & de & la \\
autoridad y y & el \\
gobierno. & &
\end{tabular} & $\begin{array}{l}\text { Hábitat, seres } \\
\text { anima- les, } \\
\text { factores } \\
\text { abióticos, tipos } \\
\text { de } \\
\text { reproducción, } \\
\text { tipos de } \\
\text { nutrición. }\end{array}$ & $\begin{array}{l}\text { La oración, el } \\
\text { párrafo, clases } \\
\text { de oración. }\end{array}$ \\
\hline $\begin{array}{l}\text { Contenidos } \\
\text { metodológicos }\end{array}$ & $\begin{array}{l}\text { Operan unes con } \\
\text { números natura } \\
\text { es y sus } \\
\text { propiedades, } \\
\text { operaciones } \\
\text { entre conjuntos, } \\
\text { faccionarios, } \\
\text { ubicación por } \\
\text { coordenadas. }\end{array}$ & $\begin{array}{l}\text { Manejo de } \\
\text { convenciones, } \\
\text { localización en - } \\
\text { mapas. }\end{array}$ & $\begin{array}{l}\text { Resolución de } \\
\text { problemas, } \\
\text { interpretación } \\
\text { de fenómenos, } \\
\text { manipulación } \\
\text { de variables. }\end{array}$ & $\begin{array}{l}\text { Lectura } \\
\text { comprensiva e } \\
\text { interpretación } \\
\text { de } \\
\text { instrucciones. }\end{array}$ \\
\hline $\begin{array}{l}\text { Contenidos } \\
\text { Valorativos }\end{array}$ & $\begin{array}{lr}\text { Producción } & \text { de } \\
\text { argumentos } & \\
\text { persuasivos } & \text { y } \\
\text { convincentes, } & \\
\text { hipótesis } & \\
\text { alrededor } & \text { de } \\
\text { soluciones } & \text { a } \\
\text { problemas } & \\
\end{array}$ & $\begin{array}{l}\text { Opinión y crítica, } \\
\text { la organización } \\
\text { interés por la } \\
\text { cultura, } \\
\text { aplicación la } \\
\text { normas y la ética }\end{array}$ & $\begin{array}{lr}\text { Conciencia } & \\
\text { sobre } & \text { el } \\
\text { ambiente y el } \\
\text { papel } & \text { del } \\
\text { hombre en } & \text { el } \\
\text { equilibrio } & \text { del } \\
\text { entorno } & \end{array}$ & $\begin{array}{l}\text { Opinión y } \\
\text { reflexión, } \\
\text { respeto por las } \\
\text { ideas. }\end{array}$ \\
\hline
\end{tabular}

— Destrezas asociadas con cada actividad propuesta en el software: 
Tabla 3. Destrezas asociadas a la mediación.

\begin{tabular}{|l|l|}
\hline $\begin{array}{l}\text { Destrezas } \\
\text { básicas }\end{array}$ & Elementos de la mediación \\
\hline Observación & $\begin{array}{l}\text { - Del entorno, de los atributos de los objetos, de los cambios, detalles } \\
\text { Diferencias y elementos específicos }\end{array}$ \\
\hline Clasificación & $\begin{array}{l}\text { - De los objetos según criterios o parámetros (palabras, animales, } \\
\text { artículos) }\end{array}$ \\
$\begin{array}{l}\text { Relaciones } \\
\text { Causa- } \\
\text { efecto }\end{array}$ & - Entre estado inicial, cambios y sugerencias \\
\hline \begin{tabular}{l} 
Inferencia \\
\hline
\end{tabular} & $\begin{array}{l}\text { - Sobre datos, hechos, premisas e in formación, gráfica, textual y } \\
\text { Numérica o cuantitativa. }\end{array}$ \\
\hline
\end{tabular}

\section{En foque pedagógico y aspectos del modelo didáctico explícito en el material:}

El programa SWINT, se diseñé dentro de un marco de referencia constructivista moderado donde las características son:

- No fragmentación de procesos, debido a que se consideran del educando los aspectos valorativos, los procesos cognitivos y las habilidades en lo procedimental. El software integrador promueve la activación de destrezas que el educando aplicará y desarrollará en forma integral para resolver las situaciones que el programa le plantea.

- Perspectiva curricular. Se asumió un modelo de integración de áreas por temas modelados como problemas reales desde situaciones cotidianas, con el propósito de crear un contexto significativo para el estudiante. Se partió de la base de que los estudiantes perciben la realidad de manera global, no segmentada.

- Preespecificación externa parcial de las actividades y contenidos. Se realizó un despliegue de contenidos (previo estudio de pertinencia y viabilidad en la integración de áreas), correspondiente a lo indicado por los Lineamientos Generales de Procesos Curriculares para el grado séptimo de básica secundaria, a través de actividades para mediar en el desarrollo de destrezas básicas. Las actividades contienen implícitos y explícitos conceptos de matemáticas, ciencias naturales, ciencias sociales y aspectos gramaticales. Algunos de los ejercicios que se proponen se actualizan de manera aleatoria. Sin embargo, se considera que el material de software es un complemento mediador, por ello, no es previsible del todo, para los diseñadores qué otras actividades o contenidos alternativos puedan desarrollarse o aprenderse en paralelo, con la actuación y guía del docente, una vez implementado el material en sesiones consecutivas.

- Simulación. Es un entorno de aprendizaje con ejercicios que simulan diferentes ambientes y situaciones dentro de un enfoque de resolución de problemas. En este caso se propone un modelo de la realidad y se reproduce. Ante la pantalla el alumno responde a una solicitud introduciendo datos a partir de los cuales la máquina ejecuta cálculos de acuerdo con el modelo que subyace en la simulación y luego muestra las modificaciones generadas por la acción del alumno. Corresponde a lo que Besnainou, Muller y Thouin (1990:62) Ilama "verdadera simulación" y se ejemplifica en el ambiente del zoológico donde los alumnos modifican los valores de las variables para producir cambios en el ambiente y restablecer la salud de los animales enfermos. 
- Flexibilidad cognitiva. El software está concebido como herramienta para el desarrollo de destrezas básicas de pensamiento desde un concepto de navegación libre y al ritmo diferencial de aprendizaje de cada educando. El alumno tiene la oportunidad de abordar aprendizajes complejos en los que intervienen distintas variables, cuya combinación puede ser diferente en función del contexto o de la situación, lo que estimula la flexibilidad cognitiva al enfrentar las mismas variables en diferentes contextos y/o enfrentar distintas variables en un mismo contexto. Además, el enfoque del software le permite recorrer la información desde puntos de vista diferentes pero teniendo siempre el estudiante la posibilidad de decidir su rumbo.

- Diseño por pareja de con textos. En la forma de presentar el material se consideró el diseño por parejas de contextos donde el rol del alumno es enteramente activo y el del docente es de facilitador. Las situaciones que se presentan en el software se encuentran enmarcadas en diferentes contextos, de manera que sea posible ejecutar acciones en un escenario de forma análoga que en otro. El estudiante deberá resolver cuáles de sus acciones se pueden transferir o aplicar a otro escenario.

Por otra parte y con base en el nivel de suficiencia alcanzado por el estudiante en el nivel de destrezas se propone paralelo al software un espacio de auto-evaluación y contraste en relación con sus logros en los procesos de observación, clasificación, relaciones, causa-efecto, inferencia, introduciendo un componente metacognitivo en el desarrollo de destrezas básicas con miras a provocar la reflexión sobre su conocimiento. (Villarini, A.1991:23-27).

Lo que se espera con el software no es transmitir un contenido, ni instruir acerca de métodos y técnicas para resolver problemas o cambiar esquemas; se provee de herramientas y recursos que el estudiante puede utilizar evaluando sus efectos y mejorando la toma de decisiones.

Considerando que los niños aprenden mejor cuando son alentados a apoyarse sobre su propia intuición y a emplear lo que ya saben para desarrollar nuevas ideas, en el proceso de diseño, se definieron los siguientes escenarios, misiones y herramientas:

- Escenarios: La ciudad, el barrio, el zoológico, el supermercado, la alcaldía, el puente y el parque, recrean en la interfaz entornos cotidianos y comunes para los educandos de séptimo grado.

- Misión 1, Misión 2 y Misión 3, representan ambientes o perspectivas distintas de los escenarios que requieren de interacción y solución de situaciones problemas con diferente nivel de dificultad.

- Herramientas: Su utilización implica acciones que realizadas por el educando sobre los escenarios generen la interacción que el sistema registrará en relación directa con las destrezas de pensamiento que se espera desarrollar a través del software, ejemplos: camión, catálogos, balde, lupa, árbol, ración, pistas, periódico, ordenanza, calculadora, manual de funciones, mapa.

Al estudiante el software le permite realizar actividades controlando libremente la cantidad de ejercicios en cada misión, observar el tiempo empleado y los resultados de su ejecución; controla el avance de una misión a otra; puede abandonar y reiniciar libremente. El software mantiene información que le apoya para resolver problemas y retos en forma de enunciados, situaciones que debe observar o ejercicios. No le indica 
cuál es la solución ni lo guía hacia las respuestas adecuadas, mantiene siempre un ambiente de libertad que propicia la reflexión y el control de las propias acciones. Además, lo vincula en línea a instrucciones u orientaciones para el manejo del programa. El software va generando un registro interno de las respuestas, las cuales marca en relación con destrezas asociadas y entrega promociones según las acciones o mensajes de alerta cuando detecta desaciertos frecuentes.

Al profesor le ofrece la posibilidad de llevar registro de los estudiantes en su interacción con el material y tener un informe como reporte del nivel de desarrollo de las destrezas (observación, clasificación, inferencia, causa-efecto). Además, le permite conocer el tiempo de ejecución de cada ejercicio. También le ofrece orientaciones sobre el significado de cada una de las destrezas que espera desarrollar con sus respectivos indicadores y recomendaciones para implementación del programa y el diseño de actividades complementarias.

En este entorno, el aprendizaje es un proceso activo en el que el individuo tiene que realizar una serie de actividades para asimilar los contenidos informativos que recibe. Según utilice o relacione la información dada para resolver ejercicios y problemas propuestos, el estudiante actúa como constructor de sus aprendizajes mediante la interacción con el entorno que le proporciona el programa y a través de la reorganización de sus esquemas de conocimiento, ya que el aprender significativamente le supone modificar los propios esquemas de conocimiento, reestructurar, revisar, ampliar y enriquecer las estructuras cognitivas.

\section{Situaciones y modelo de evaluación:}

El programa SWINT en la medida en que provee actividades para ejercitarse en las destrezas básicas determina por contrastación frente a los indicadores, el nivel de desarrollo de las mismas. También guarda un registro del tiempo que ocupa el estudiante para resolver los ejercicios en cada misión y en cada escenario. Se considera que la duración es un parámetro que revela la eficiencia en la aplicación de la destreza.

El sistema de evaluación está relacionado con todos los ejercicios realizados en cada escenario del micromundo. De acuerdo con esto y definido el nivel de logro para cada misión, unido a todas las características, nivel de dificultad, tipo de destreza, etc., en el programa se evalúa qué ha hecho el usuario en el entorno de las actividades y si lo hizo correctamente o no. Estos logros se registran en la base de datos que el programa tiene y arroja informes impresos o por pantalla individuales o por grupos.

La relación entre escenarios modelados, los tipos de ejercicios y la forma de interacción-operación en el entorno mediático se visualiza en la tabla 1 de la página siguiente.

En función del momento de evaluación existen varios tipos de evaluación aplicadas en este programa de software educativo:

- Prueba de entrada: aplicada antes de iniciar la interacción con los escenarios propuestos en el software, para que el profesor realice un aprestamiento con la mediación y genere en el estudiante el interés y la motivación por el tipo de ambiente y manipulación que deberá aplicar para resolver las misiones de cada escenario. Al intentar entrar a los escenarios, se genera una lista de 3 opciones de pruebas de entrada que pretenden hacerlo observar y clasificar o rotular objetos (animales o cosas) según atributos o propie- 
dades; el ambiente de la mediación es similar al que el estudiante está acostumbrado en los textos impresos: debe seleccionar de una lista las características o atributos de los objetos que aleatoriamente se le proponen en casillas (prueba 1 el zoológico, prueba 2 el parque, prueba 3 el supermercado). Si el estudiante supera 30 respuestas correctas se activan en la interfaz todos los accesos a la misión 1, 2 y 3 de cada escenario.

- Evaluación sumativa: se puede acceder desde el módulo del profesor a cualquier altura del programa para averiguar los logros de cada aprendiz con respecto al nivel de desempeño en cada destreza. Se pueden ver también resultados de la evaluación por grupo, lo que permite comparar el grupo consigo mismo en las diferentes sesiones de aplicación del software.

- Evaluación formativa y retroalimentación: después de situaciones para ayudar a descubrir o practicar, transferir y afianzar destrezas o habilidades, el estudiante usuario verá en un acumulado sus avances en forma de puntaje; además, recibe orientación a través de mensajes de instrucción motivadores, algunos, orientadores y de alerta otros, que le permiten establecer si ha cometido errores o le estimulará si ha desarrollado un buen trabajo.

Desde este punto de vista, se puede decir que en el software se implementa una evaluación:

- Implícita, cuando el estudiante detecta sus errores, se evalúa, a partir de las respuestas y estímulos o señales que le da el programa.

- Explícita, cuando el programa presenta informes valorando la actuación del alumno.

Para decidir si el usuario ha logrado determinado nivel de aprendizaje se establecieron criterios claros. Todos los elementos del modelo computacional del micromundo en forma de iconos sensibles reaccionan ante determinados eventos. Estos eventos se modelaron especificando destrezas asociadas con acciones del usuario. También se especificó, para cada elemento de los mapas sensibles y objetos de la pantalla, ante cuáles eventos está en capacidad de reaccionar acumulando puntos en las destrezas según el modelo relacional diseñado para la base de datos del programa computacional. 
Estructura básica del software

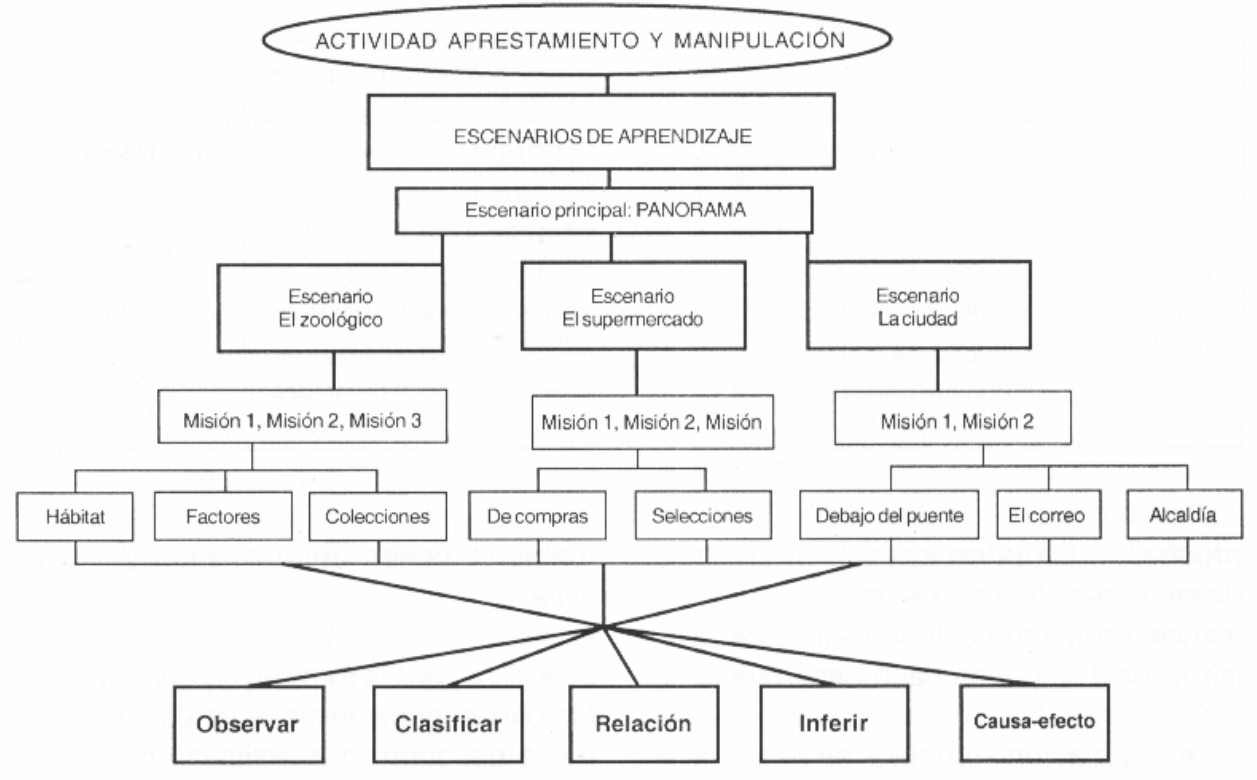

Gráfico 1. Estructura básica del software educativo SWINT-DBP.

Como se puede apreciar en este esquema, el software tiene inicialmente actividades cuya finalidad es el aprestamiento y la familiarización con la mediación, además de apoyar en establecer si el estudiante tiene los requisitos mínimos para desarrollar los contenidos asociados a cada misión en cada escenario donde las tareas se presentan como estrategias didácticas que el sistema administra y tienen diseño variado en cuanto a dificultad y nivel de logros, conservando equivalencia en las actividades en los diferentes escenarios, con el propósito de llevar la transferencia en las destrezas; el estudiante, a medida que ejecuta acciones asociadas con los indicadores o usa herramientas previstas para desarrollar el nivel, puede ir verificando el tipo de cambios.

\section{Requerimientos del usuario para el uso del software}

El estudiante debe tener un mínimo desempeño en el uso del mouse porque muchas de las decisiones a tomar en él las debe realizar haciendo clic, y, un reconocimiento de las ventanas de Windows: abrir, cerrar ventanas, entrar, salir, navegar, iconos, botones, menús y herramientas etc, para así facilitar el desplazamiento dentro de ellas. Para tener un buen desempeño y alcanzar los objetivos propuestos, se le recomienda al usuario alumno o profesor que haga uso de toda la información que le presenta el software en Ayudas, en el Manual y la que aparece en pantalla al pasar el mouse. El programa presenta una ventana principal con tres zonas sensibles: estudiantes, profesor y créditos, haciendo clic en cualquiera de ellas tiene acceso a pantallas distintas, sólo que para acceder a la opción del profesor requiere de una clave o Password.

Para realizar libremente el recorrido por la ciudad, el estudiante debe obtener un mínimo de 30 puntos en cualquiera de las pruebas de aprestamiento.

Todas las opciones del software están documentadas por una ayuda muy breve y precisa, es necesario que se motive el estudiante a consultarlas para que se informe acerca de las acciones que va a realizar y no navegue adivinando. Además, así se incentiva la actividad 
lectora de texto, de símbolos e imágenes requerida frente a la cantidad de información disponible representada en el sistema y se ejercita la atención para lograr la comprensión y el seguimiento de sugerencias e instrucciones. Cada pantalla que aparece, deja claro cuál es el rol que el usuario juega, las herramientas de interacción que podrá usar y le permite apreciar el reto que debe resolver mientras ejercita sus destrezas.

\section{Requerimientos técnicos:}

\begin{tabular}{|c|c|}
\hline ESPECIFICACIONES & DETALLE \\
\hline Sistema Operativo & Windows 98,2000 o Milenium \\
Procesador & $266 \mathrm{Mhz}$ o superior \\
Pantalla y tarjeta graficadora & Resolución $640 \times 480,16$ bits \\
Espacio disponible en el disco & $50 \mathrm{Mb}$ \\
Unidad de CD & Mínimo 4x de velocidad de reproducción \\
Memoria RAM & $64 \mathrm{Mb}$ \\
Mouse & $\mathrm{Ps} / 2$ \\
\hline
\end{tabular}

\section{Consideraciones finales}

El prototipo del software se probó con dos grupos de estudiantes de séptimo grado, de cuya experiencia se pueden advertir las siguientes consideraciones.

- La causa probable de la falta de ejercicio en algunos de los escenarios está asociada con elementos del diseño computacional o de la interfaz, por ejemplo, la presencia de estímulos en algunas de las actividades más no en todo el contexto de la mediación.

- Al no exigirse un recorrido determinado por el profesor, algunos estudiantes se limitan a realizar las actividades que le generan más lúdica que esfuerzo cognitivo.

- Las dificultades expresadas en la comprensión de algunas orientaciones del software denotaron el escaso hábito y gusto por la lectura, a pesar de que el diseño se preocupó por la dosificación de los textos y su despliegue económico en la pantalla.

Se realizó, igualmente una evaluación del software con un diseño cuasiexperimental con grupo de control con dos grupos de estudiantes: uno de Montería y otro de Barranquilla. Se aplicó el estadístico t de student para valorar las diferencias de medias entre los grupos en cada una de las destrezas estudiadas, encontrándose diferencias significativas a favor de los grupos experimentales en la mayoría de las destrezas. En la actualidad se prepara un artículo sobre el proceso de investigación llevado a cabo con su correspondiente análisis y discusión de resultados. En esta ponencia se hace énfasis en el desarrollo y probación de la herramienta.

Usar este software como soporte, como estrategia de mediación y representación de entornos, adquiere una dimensión más profunda si la intervención es ideada pedagógicamente, sobre la base de un concepto de currículo y desarrollo humano, que si sólo se tiene la intención de modelar aplicaciones informáticas para aprovechar las bondades y ventajas que representa el computador como dispositivo instrumental útil en el tratamiento, presentación y organización de la información o como factor de motivación. Esto último es lo que sustenta la mayoría de las iniciativas de incorporación de tecnologías al currículo escolar, descuidándose la indagación y prueba de hipótesis sobre el papel que juegan las mediaciones computarizadas en el cambio cognitivo, lo mismo que 
las posibles relaciones entre el desarrollo de las dimensiones humanas y la formulación e implementación sistemática de instrumentos tecnológicos pensados como didáctica interactiva de procesos integrales, intelectuales, volitivos y actitudinales.

Durante la probación del software se realizaron permanentemente observaciones y registros del protocolo en diario de campo y videos, lo que permitió encontrar una serie de implicaciones favorables, las que sistematizadas a continuación se pueden mirar desde diferentes abordajes:

- Desde la perspectiva de los contenidos: el software integrador, utilizado como mediación, incrementa el contacto natural y espontáneo con temas diversos, sin importar la naturaleza y origen de los objetos de conocimiento, utilizando los contenidos conceptuales, procedimentales y actitudinales integrados de las áreas del plan de estudio (matemáticas, ciencias naturales, lenguaje y sociales), como sustratos de información sobre las que el educando puede operar y aplicar sus estrategias desarrollando destrezas desde un nivel literal de pensamiento que le implica en primera instancia leer y comprender, hasta uno más inferencial que lo lleva a aplicar el contenido y hallar el sentido de los aprendizajes.

- Desde la perspectiva de la enseñabilidad: es más que una actividad de implementación/programación didáctica de contenidos y desarrollo de interíaces de computador; es un avance en el modeamiento de las plataformas de interacción, concebidas como zonas de desarrollo próximo a partir de a comprensión misma y representación funcional del conocimiento que el maestro tiene del contenido que enseña y no sólo desde lo que aparece reportado en los textos guía de los que habitualmente se vale para preparar sus clases.

- Desde la mirada de los estudiantes: El software Swint-DBP constituye una invitación para el uso de los conceptos previos y la movilización de lo aprendido; por medio de la manipulación de contenidos, representados o explícitos en las metáforas de la aplicación computacional se favorece la valoración de la información y la búsqueda de sentido de la misma en el contexto que se propone en cada escenario. Además, para el estudiante, el poder expresar lo que hace y sustentar por qué lo hace y verificar qué pasa si no lo hace, es una dimensión valiosa de la metacognicíón poco explorada y menos aprovechada en su potencial educativo, que se descubre en el marco de la implementación de este software para el desarrollo de destrezas básicas.

- Desde el papel del computador y el software educativo: en el ambiente de aprendizaje como entorno mediatizador, las simulaciones y representaciones son favorecedoras del acercamiento a temas y problemas no posibles de manejar interactivamente en la cotidianidad; se propicia, la aplicación y transferencia de estrategias para resolver tareas, la toma de conciencia, el control y la transformación de roles, la autonomía, el entusiasmo, el reto y la iniciativa. Estos fueron valores en cuyo ámbito se percibió mejor el progreso en las destrezas. Una ganancia realmente valiosa pues generalmente el uso de software en las salas de informática de los centros educativos ha sido asociado por los niños con el juego y el entretenimiento, pero no se percatan de su "alor en el desarrollo de la mente dado que en el contexto escolar tradicional ha sido dejado de lado por los educadores especializados en informática educativa, el aprovechar la posibilidad de educar con tecnología para el desarrollo de competencias en lo interactivo y lo representacional, como factor de éxito en aprendizajes más duraderos, más significativos, más autónomos o autocontrolados, asistidos por computador. 
- Desde la investigación y desarrollo en informática: la ideación y uso de aplicaciones computacionales para enseñar a aprender o enseñar a pensar o para hacerse conscientes de las implicaciones individuales del aprendizaje, es un campo prolífico que en Colombia promete la generación de modelos apropiados, a partir de la indagación de contextos y la experimentación comprometida con la expansión creativa de experiencias para transformar la realidad en términos de la calidad de los ambientes escolares. Compromiso que se extiende a la construcción de una pedagogía renovada a tono con las nuevas tendencias en el ámbito comunicacional y las actuales exigencias de los actores educativos.

Aunque se podría pensar que el diseño de materiales educativos computacionales entró en una etapa de vertiginosa evolución hacia modelos de inteligencia artificial o de sistemas sofisticados de simulación y ejercitación con base en juegos, con poderosas interfases, no se puede perder de vista que el software desarrollado institucionalmente en cumplimiento de procesos de investigación educativa tiene una función de contexto válida para el tratamiento de las particulares características de los educandos en Colombia.

\section{Bibliografía}

Alanis Huerta, A. (2000) "La tecnología educativa: entre el saber y el hacer". Revista digital Contexto Educativo, Número 5 -Marzo de 2000.

Besnainou, R.; Muller, C. y Thouin, C. (1990). Cómo elaborar programas interactivos. Barcelona, CEAC.

De Zubiría, Miguel (1995) Fundamentos de Pedagogía Conceptual. Bogotá, Plaza y Janés.

Fogarty, R. (1999) “Architects of the Intellect”. Educational Leadership. Vol. 57, No. 3.

Gros, Begoña (1997) Diseños y programas Educativos: pautas pedagógicas para la elaboración de software. Barcelona, Editorial Ariel.

Merrill, 0. (1991). Constructivism and instructional design. Educational Tech nology, mayo, pp. 45-52.

Perkins, D. (1991). Technology metes constructivism: Do they make marriage? Educational Technology. Mayo, pp. 18-23.

Villarini, Ángel. (1991) Manual para la enseñanza de las destrezas de pensamiento. Puerto Rico, Pell. 